\title{
A NOTE ON THE WEISS CONJECTURE
}

\author{
NICK GILL
}

(Received 18 March 2013; accepted 14 May 2013; first published online 7 August 2013)

\author{
Communicated by B. Alspach
}

\begin{abstract}
Let $G$ be a finite group acting vertex-transitively on a graph. We show that bounding the order of a vertex stabiliser is equivalent to bounding the second singular value of a particular bipartite graph. This yields an alternative formulation of the Weiss conjecture.
\end{abstract}

2010 Mathematics subject classification: primary 20B25; secondary 05 C25.

Keywords and phrases: permutation group, vertex-transitive, singular value, Weiss conjecture.

\section{Introduction}

Throughout this note, $G$ is a finite group acting vertex-transitively on a graph $\Gamma=(V, E)$ of valency $k$. We say that $G$ is locally-P, for some property $\mathrm{P}$, if $G_{v}$ is $\mathrm{P}$ on $\Gamma(v)$. Here $v$ is a vertex of $\Gamma$, and $\Gamma(v)$ is the set of neighbours of $v$. With this notation we can state the Weiss conjecture [9].

Conjecture 1 (The Weiss conjecture). There exists a function $f: \mathbb{N} \rightarrow \mathbb{N}$ such that if $G$ is vertex-transitive and locally-primitive on a graph $\Gamma$ of valency $k$, then $\left|G_{v}\right|<f(k)$.

A stronger version of this conjecture, in which 'primitive' is replaced by 'semiprimitive' has been recently proposed [6]. (A transitive permutation group is said to be semiprimitive if each of its normal subgroups is either transitive or semiregular.)

Our aim in this note is to connect the order of $G_{v}$ to the singular value decomposition of the biadjacency matrix of a particular bipartite graph $\mathcal{G}$. This connection yields an alternative form of the Weiss conjecture (and its variants). Our main result is the following theorem (we write $\lambda_{2}$ for the second largest singular value of the biadjacency matrix of $\mathcal{G}$ ).

THEOREM 1.1. For every function $f: \mathbb{N} \rightarrow \mathbb{N}$, there is a function $g: \mathbb{N} \rightarrow \mathbb{N}$ such that if $G$ is a finite group acting vertex-transitively on a graph $\Gamma=(V, E)$ of valency $k$ and $\lambda_{2}<f(k)$, then $\left|G_{v}\right|<g(k)$.

(C) 2013 Australian Mathematical Publishing Association Inc. 1446-7887/2013 \$16.00 
Conversely, for every function $g: \mathbb{N} \rightarrow \mathbb{N}$, there is a function $f: \mathbb{N} \rightarrow \mathbb{N}$ such that if $G$ is a finite group acting vertex-transitively on a graph $\Gamma=(V, E)$ of valency $k$ and $\left|G_{v}\right|<g(k)$, then $\lambda_{2}<f(k)$.

All of the necessary definitions pertaining to Theorem 1.1 are discussed below. In particular, the bipartite graph $\mathcal{G}$ is defined in Section 1 , and the singular value decomposition of its biadjacency matrix is discussed in Section 2.

Theorem 1.1 implies that, to any family of vertex-transitive graphs with bounded vertex stabiliser, we have an associated family of bipartite graphs with bounded second singular value, and vice versa. Proving the Weiss conjecture (or one of its variants) is, therefore, equivalent to bounding the second singular value for a particular family of bipartite graphs.

Gowers remarks that singular values are the "correct analogue of eigenvalues for bipartite graphs' (see the preamble to Lemma 2.7 in [4]) ${ }^{1}$. Thus bounding the second singular value of a bipartite graph is analogous to bounding the second eigenvalue of a graph; the latter task is a celebrated and much studied problem due to its connection to the expansion properties of a graph (see, for instance, [5]).

The fact that the Weiss conjecture has connections to expansion has already been recognised [7], we hope that this note adds to the evidence that it is a connection warranting a good deal more investigation.

\section{The associated bipartite graph $G$}

Our first job is to describe $\mathcal{G}$, and for this we need the concept of a coset graph. Let $H$ be a subgroup of $G$ and let $A$ be a union of double cosets of $H$ in $G$ such that $A=A^{-1}$. Define the coset graph $\operatorname{Cos}(G, H, A)$ as the graph with vertex set the left cosets of $H$ in $G$ and with edges the pairs $\{x H, y H\}$ such that $H x^{-1} y H \subset A$. Observe that the action of $G$ by left multiplication on the set of left cosets of $H$ induces a vertex-transitive automorphism group of $\operatorname{Cos}(G, H, A)$.

The following result is due to Sabidussi [8].

Proposition 2.1. Let $\Gamma=(V, E)$ be a $G$-vertex-transitive graph and $v$ a vertex of $\Gamma$. Then there exists a union $S$ of $G_{v}$-double cosets such that $S=S^{-1}, \Gamma \cong \operatorname{Cos}\left(G, G_{v}, S\right)$ and the action of $G$ on $V$ is equivalent to the action of $G$ by left multiplication on the left cosets of $G_{v}$ in $G$.

Note that $G$ is locally-transitive if and only if $S$ is equal to a single double coset of $G_{v}$. From here on we fix $v$ to be a vertex in $V$ and we set $S$ to be the union of double cosets of $G_{v}$ in $G$ such that $\Gamma \cong \operatorname{Cos}\left(G, G_{v}, S\right)$. Observe that $S(\{v\})=\Gamma(v)$.

\footnotetext{
${ }^{1}$ The mathematics behind this remark is set down in [2]. An elementary first observation is that the eigenvalues of the natural biadjacency matrix of a bipartite graph may be negative, in contrast to the eigenvalues of the (symmetric) adjacency matrix of a graph. This pathology is remedied by studying the singular values as we shall see.
} 
We are ready to define the regular bipartite graph $\mathcal{G}$. We define the two vertex sets, $X$ and $Y$, to be copies of $V$. The number of edges between $x \in X$ and $y \in Y$ is defined to equal the number of elements $s \in S$ such that $s(x)=y$. Note that $\mathcal{G}$ is a multigraph.

\section{The singular value decomposition}

For $V$ and $W$ two real inner product spaces, we define a linear map

$$
w \otimes v: V \rightarrow W, x \mapsto\langle x, v\rangle w .
$$

With this notation we have the following result [4, Theorem 2.6].

Proposition 3.1. Let $\alpha: V \rightarrow W$ be a linear map. Then $\alpha$ has a decomposition of the form $\sum_{i=1}^{k} \lambda_{i} w_{i} \otimes v_{i}$, where the sequences $\left(v_{i}\right)$ and $\left(w_{i}\right)$ are orthonormal in $V$ and $W$, respectively, each $\lambda_{i}$ is nonnegative, and $k$ is the smaller of $\operatorname{dim} V$ and $\operatorname{dim} W$.

The decomposition described in the proposition is called the singular value decomposition, and the values $\lambda_{1}, \lambda_{2}, \ldots$ are the singular values of $\alpha$. In what follows we always assume that the singular values are written in nonincreasing order: $\lambda_{1} \geqslant \lambda_{2} \geqslant \cdots$.

Now write $\mathcal{A}$ for the biadjacency matrix of $\mathcal{G}$ as a bipartite graph, that is, the rows of $\mathcal{A}$ are indexed by $X$, the columns by $Y$ and, for $x \in X, y \in Y$, the entry $\mathcal{A}(x, y)$ is equal to the number of edges between $x$ and $y$. Then $\mathcal{A}$ can be thought of as a matrix for a linear map $\alpha: \mathbb{R}^{X} \rightarrow \mathbb{R}^{Y}$ and, as such, we may consider its singular value decomposition. From here on the variables $\lambda_{1}, \lambda_{2}, \ldots$ will denote the singular values of this particular map.

The next result gives information about this decomposition. (The result is [3, Lemma 3.3], although some of the statements must be extracted from the proof.)

Lemma 3.2. (1) We have $\lambda_{1}=t \sqrt{\left|V_{1}\right|\left|V_{2}\right|}$, where $t$ is the real number such that every vertex in $V_{1}$ has degree $t\left|V_{2}\right|$.

(2) If $f$ is a function that sums to zero, then $\|\alpha(f)\| /\|f\| \leqslant \lambda_{2}$.

Note that the only norm used in this note is the $\ell^{2}$-norm.

\section{Convolution}

Consider two functions $\mu: G \rightarrow \mathbb{R}$ and $\nu: V \rightarrow \mathbb{R}$. We define the convolution of $\mu$ and $v$ to be

$$
\mu * v: V \rightarrow \mathbb{R}, \quad v \mapsto \sum_{g \in G} \mu(g) v\left(g^{-1} v\right) .
$$

In the special case where $\mu=\chi_{S}$, the characteristic function of the set $S$ defined above, $\chi_{S} * v$ takes on a particularly interesting form:

$$
\left(\chi_{S} * f\right)(v)=\sum_{g \in G} \chi_{S}(g) f\left(g^{-1} v\right)=\sum_{w \in V} \mathcal{A}(v, w) f(v) .
$$


Here, as before, $\mathcal{A}$ is the biadjacency matrix of the bipartite graph $\mathcal{G}$. Equation (1) implies that the linear map $\alpha: \mathbb{R}^{X} \rightarrow \mathbb{R}^{Y}$, for which $\mathcal{A}$ is a matrix, is given by $\alpha(f)=\chi_{S} * f$. This form is particularly convenient, as it allows us to use the following easy identities [3, Lemma 2.3].

LeMma 4.1. Let $f$ be a function on $V$ that sums to $0, p$ a probability distribution over $V$, q a probability distribution over $G$, and $U$ the uniform probability distribution over $V$. Then:

(1) $\|f+U\|^{2}=\|f\|^{2}+1 /|V|$;

(2) $\quad\|p-U\|^{2}=\|p\|^{2}-1 /|V|$;

(3) $\|q *(p \pm U)\|=\|q * p \pm U\|$;

(4) for $k$ a real number, $\|k p\|=k\|p\|$.

\section{The proof}

Theorem 1.1 will follow from the next result, which shows that, provided $k$ is not too large compared to $|V|$, the order of $G_{v}$ is bounded in terms of $\lambda_{2}$ and $k$.

Proposition 5.1. Either $\left|G_{v}\right|<\sqrt{2} \lambda_{2} / k$ or $|V|<2 k$.

Proof. Let $v$ be a vertex in $V$. We define two probability distributions, $p_{S}: G \rightarrow \mathbb{R}$ and $p_{v}: V \rightarrow \mathbb{R}$, as follows:

$$
p_{S}(x)=\left\{\begin{array}{ll}
\frac{1}{|S|} & x \in S, \\
0 & x \notin S,
\end{array} \quad p_{v}(x)= \begin{cases}1 & x=v \\
0 & \text { otherwise }\end{cases}\right.
$$

Observe that $\left\|p_{S}\right\|=1 / \sqrt{|S|}=1 / \sqrt{k\left|G_{v}\right|}$ and $\left\|p_{v}\right\|=1$. Observe that $\left(p_{S} * p_{v}\right)(w)=$ 0 except when $w \in S(\{v\})=\Gamma(v)$. A simple application of the Cauchy-Schwarz inequality (or see [1, Observation 3.4]) gives

$$
\frac{1}{k}=\frac{1}{|\Gamma(v)|} \leqslant\left\|p_{S} * p_{v}\right\|^{2} .
$$

Define $f=p_{v}-U$ and observe that $f$ is a function on $V$ that sums to 0 . Lemma 3.2 implies that $\|(\alpha f)\| /\|f\| \leqslant \lambda_{2}$. Using this fact, the identities in Lemma 4.1, and the fact that $\chi_{S}=|S| p_{S}$, we obtain

$$
\begin{aligned}
\frac{1}{k} & \leqslant\left\|p_{S} * p_{v}\right\|^{2} \\
& =\left\|p_{S} *(f+U)\right\|^{2} \\
& =\left\|p_{S} * f+U\right\|^{2} \\
& =\left\|p_{S} * f\right\|^{2}+\frac{1}{|V|} \\
& =\frac{1}{|S|^{2}}\left\|\chi_{S} * f\right\|^{2}+\frac{1}{|V|}
\end{aligned}
$$




$$
\begin{aligned}
& =\frac{1}{|S|^{2}}\|\alpha(f)\|^{2}+\frac{1}{|V|} \\
& \leqslant \frac{1}{|S|^{2}} \lambda_{2}^{2}\|f\|^{2}+\frac{1}{|V|} \\
& =\frac{1}{|S|^{2}} \lambda_{2}^{2}\left\|p_{v}-U\right\|^{2}+\frac{1}{|V|} \\
& <\frac{\lambda_{2}^{2}}{|S|^{2}}+\frac{1}{|V|} .
\end{aligned}
$$

Since $|S|=k\left|G_{v}\right|$ we can rearrange to obtain

$$
k>\frac{|V|}{1+\frac{\lambda_{2}^{2}|V|}{k^{2}\left|G_{V}\right|^{2}}} .
$$

Observe that if $\lambda_{2}^{2}|V| / k^{2}\left|G_{v}\right|^{2} \leqslant 1$, then

$$
k>\frac{|V|}{1+\frac{\lambda_{2}^{2}|V|}{k^{2}\left|G_{v}\right|^{2}}} \geqslant \frac{|V|}{2}
$$

and the result follows. On the other hand, if $\lambda_{2}^{2}|V| / k^{2}\left|G_{v}\right|^{2}>1$, then

$$
k>\frac{|V| k^{2}\left|G_{v}\right|^{2}}{k^{2}\left|G_{v}\right|^{2}+|V| \lambda_{2}^{2}}>\frac{|V| k^{2}\left|G_{v}\right|^{2}}{2|V| \lambda_{2}^{2}}
$$

and we conclude that $\left|G_{v}\right|^{2}<2 \lambda_{2}^{2} / k$ as required.

Finally we can prove Theorem 1.1.

Proof of Theorem 1.1. The previous lemma implies that if $\lambda_{2}<f(k)$ for some function $f: \mathbb{N} \rightarrow \mathbb{N}$ then $\left|G_{v}\right|<g(k)$ for some function $g: \mathbb{N} \rightarrow \mathbb{N}$. (Note that if $|V| \leqslant 2 k$, then $\left|G_{v}\right| \leqslant|G| \leqslant(2 k) !$.)

For the converse, Lemma 3.2 implies that $\lambda_{1}=t \sqrt{|X| \cdot|Y|}$, where $t$ is the real number such that every vertex in $X$ has degree $t|Y|$. Now recall that $|X|=|Y|=|V|$ and observe that every vertex in $X$ has degree $k\left|G_{v}\right|$. Thus we conclude that $\lambda_{1}=k\left|G_{v}\right|$. Since $\lambda_{2} \leqslant \lambda_{1}$ the result follows.

\section{References}

[1] L. Babai, N. Nikolov and L. Pyber, 'Product growth and mixing in finite groups', in: Proceedings of the Nineteenth Annual ACM-SIAM Symposium on Discrete Algorithms (New York) (ACM, 2008), 248-257.

[2] B. Bollobás and V. Nikiforov, 'Hermitian matrices and graphs: singular values and discrepancy', Discrete Math. 285(1-3) (2004), 17-32.

[3] N. Gill, 'Quasirandom group action', J. Eur. Math. Soc. (JEMS), to appear. arXiv:1302.1186.

[4] W. T. Gowers, 'Quasirandom groups', Combin. Probab. Comput. 17 (2008), 363-387. 
[5] A. Lubotzky, 'Discrete groups, expanding graphs and invariant measures', in: Modern Birkhäuser Classics (Birkhäuser, Basel, 2010), with an appendix by Jonathan D. Rogawski, reprint of the 1994 edition.

[6] P. Potočnik, P. Spiga and G. Verret, 'On graph-restrictive permutation groups', J. Combin. Theory Ser. B 102(3) (2012), 820-831.

[7] C. Praeger, L. Pyber, P. Spiga and E. Szabó, 'Graphs with automorphism groups admitting composition factors of bounded rank', Proc. Amer. Math. Soc. 140(7) (2012), 2307-2318.

[8] G. Sabidussi, 'Vertex-transitive graphs', Monatsh. Math. 68 (1964), 426-438.

[9] R. Weiss, 's-transitive graphs', in: Algebraic Methods in Graph Theory, Vol. I, II (Szeged, 1978), Vol. 25, Colloq. Math. Soc. János Bolyai (North-Holland, Amsterdam, 1981), 827-847.

NICK GILL, Department of Mathematics, The Open University,

Walton Hall, Milton Keynes, MK7 6AA, UK

e-mail: n.gill@open.ac.uk 